LAWRENCE LIVERMORE NATIONAL LABORATORY

\title{
Sheath Physics and \\ Boundary Conditions for Edge Plasmas
}

R. H. Cohen and D. D. Ryutov

September 3, 2003

Plasma Edge Theory Conference, San Diego, California, September 3-5, 2003 


\section{DISCLAIMER}

This document was prepared as an account of work sponsored by an agency of the United States Government. Neither the United States Government nor the University of California nor any of their employees, makes any warranty, express or implied, or assumes any legal liability or responsibility for the accuracy, completeness, or usefulness of any information, apparatus, product, or process disclosed, or represents that its use would not infringe privately owned rights. Reference herein to any specific commercial product, process, or service by trade name, trademark, manufacturer, or otherwise, does not necessarily constitute or imply its endorsement, recommendation, or favoring by the United States Government or the University of California. The views and opinions of authors expressed herein do not necessarily state or reflect those of the United States Government or the University of California, and shall not be used for advertising or product endorsement purposes.

This is a preprint of a paper intended for publication in a journal or proceedings. Since changes may be made before publication, this preprint is made available with the understanding that it will not be cited or reproduced without the permission of the author. 


\title{
Sheath Physics and Boundary Conditions for Edge Plasmas
}

\author{
R. H. Cohen* and D.D. Ryutov \\ L-630, Lawrence Livermore National Laboratory, Livermore, CA 94551 USA
}

Received 3 September 2003

Key words boundary conditions,edge, sheath, plasma.

PACS 52.55.Rk;52.25Fi;52.55Ez

The boundary conditions of mass, momentum, energy, and charge appropriate for fluid formulations of edge plasmas are surveyed. We re-visit the classic problem of 1-dimensional flow, and note that the "Bohm sheath criterion" is requirement of connectivity of the interior plasma with the external world, not the result of termination of the plasma by a wall. We show that the nature of the interior plasma solution is intrinsically different for ion sources that inject above and below the electron sound speed. We survey the appropriate conditions to apply, and resultant fluxes, for a magnetic field obliquely incident on a wall, including the presence of drifts and radial transport. We discuss the consequences of toroidal asymmetries in wall properties, as well as experimental tests of such effects. Finally, we discuss boundary-condition modifications in the case of rapidly varying plasma conditions.

\section{Introduction}

The topic of boundary conditions for a plasma impacting on a wall is one of the oldest in plasma physics, The notion of a plasma transitioning into a sheath region dates back at least to Langmuir [1,2]. Beginning with the explicit formulation of the sheath criterion by Bohm [3], there have been many papers developing the theory for collisionles and collisional plasmas; see, e.g. the review of Riemann [4] and the many references therein. Also a very nice survey of the physics ingredients of the canonical picture that has emerged (along with nice surveys of most other topics in boundary plasmas!) can be found in Stangeby's book [5].

More recently, studies have been extended to include the case of oblique incidence of magnetic field lines on walls, first by Chodura [6], with subsequent extensions to incorporate the effects of drifts [7, 8, 9], collisions $[13,15,17]$ and effects of radial drifts[9].

Despite this intense effort, there remain open questions, even in the simplest cases of one-dimensional collisionless and collisional flows. We survey this case in Sec. 2 and note that some commonly held beliefs, such as (1) that satisfying the Bohm criterion is the result of the existence of a Debye sheath at the wall, (2) that acceleration necessarily occurs in a source region, and (3) that for a sub-sonic source, a potential drop with a spatial extent comparable to that of the source and of a size just sufficient to produce sonic flow occurs, are not always true. In particular, the Bohm criterion emerges as a requirement of connectivity of the interior solution with the external world, apparently independent of the specific incarnation of "external world": the same conditions must be met, for example, for an interior plasma solution to connect to an open-ended expanding flux tube. Also there are essential differences between solutions for sources that inject below the sound speed and those that inject above.

In Sec. 3 we consider the situation of most common interest for magnetic-fusion edge plasmas, which is a magnetic field obliquely incident on a plasma, and review the boundary conditions for steady mass, current, and energy flows to bounding surfaces, including effects of drifts, radial transport, and applied bias potentials. The boundary conditions so presented offer the possibility of influencing the scrape-off layer plasma by introducing toroidal variations in properties of bounding surfaces $[19,20]$; the theoretical arguments, along with experimental

* Corresponding author: e-mail: rcohen@IInl.gov, Phone: 1925422 9831, Fax: 9254233484 
evidence of the predicted effects, is discussed in Sec. 4. In Sec. 5 we discuss modfications to the boundary conditions for time-varying plasma conditions; these corrections can be appreciable for high-frequency fluctuations. Finally, concluding remarks and a summary are offered in Sec. 6.

\section{Normal Incidence}

In this section, we discuss the classical problem of boundary conditions for the case where the magnetic field intersects an absorbing wall at a right angle. We will not review the rich history of research in this area (which was summarized some time ago by Riemann [4]) but will rather present a discussion of several aspects of this problem that are relatively little known. For the most part, we concentate on the collisionless situation but make also a few brief comments on the collisional case. In our discussion we assume that the global scale of the system $L$ (say, the connection length in a tokamak SOL) is much greater than the Debye length $\lambda_{D}$.

\subsection{Elementary analysis of sheath}

The standard analysis of the sheath proceeds along the following lines (see, e.g., [4]): Consider a uniform stream of ions all having the same velocity $u$ along the magnetic field. Electrons will be assumed Boltzmann, with temperature $T_{e}$. When such a uniform neutral plasma flow approaches the wall, a non-quasineutral sheath repelling the majority of the electrons must be formed (Fig. 1). In other words, a substantial drop of the potential occurs near the wall, from $\Phi=0$ in the incoming flow (this is our choice of the reference point for the potential) to

$$
\Phi=\Phi_{w} \approx-\Lambda T_{e} / e
$$

where $\Lambda$ is of order $\ln \left(m_{e} / m_{i}\right)$ (cf. [5]).

If we consider the asymptotic region circled in Fig. 1, where $\Phi$ hasn't changed much from its zero value, it is obvious from the figure that $\Phi^{\prime \prime}$ must be negative, where prime means $d / d z$. Hence Poisson's equation, $\Phi^{\prime \prime}=4 \pi e\left(n_{e}-n_{i}\right)$, tells us that $n_{i}-n_{e}$ must be greater than zero. But also in this region, because the problem is one-dimensional, the electron and ion densities can be given as an expansion in powers of $\Phi$, yielding, in leading order, $\left.n_{i, e} \approx \Phi\left(\partial n_{i, e} / \partial \Phi\right)\right|_{\Phi=0}$. Since $\Phi<0$, one immediately sees that a smooth stationary transition to a sheath is possible only if

$$
\left(\left.\frac{\partial n_{i}}{\partial \Phi}\right|_{\Phi=0}-\left.\frac{\partial n_{e}}{\partial \Phi}\right|_{\Phi=0}\right)<0
$$

This condition is often called "the Bohm condition," although Bohm formulated an analogous condition in an equality form.

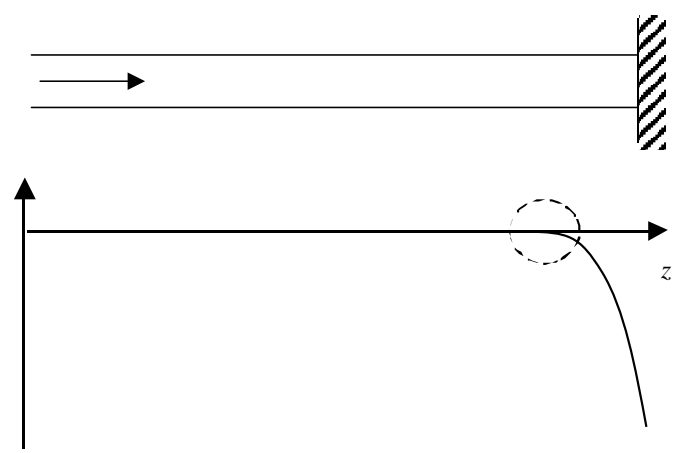

Fig. 1 Plasma flow towards absorbing wall. The sources are situated to the left, outside the boundaries of the figure. In the encircled area, the potential is close to zero, while its second derivative is negative.
In a model with monoenergetic ions and Boltzmann electrons, the densities depend on the potential as $n_{i}=n_{0}(1-2 \phi / \eta)^{-1 / 2} ; n_{e}=n_{0} \exp \phi$, where $\phi=e \Phi / T_{e}, n_{0}=n_{i}(\Phi=0)=n_{e}(\Phi=$ $0)$, and $\eta=M u^{2} / T_{e}$ In this case the Bohm condition becomes, simply, $\eta>1$. We conclude that in this case which we call the "supercritical case", the formation of a monotonic Debye sheath is possible.

However, in the opposite case of $\eta<1$ there are no reasonable solutions to Poisson's equation satisfying boundary conditions infinitely far from the wall ( $\Phi \rightarrow 0, \Phi^{\prime} \rightarrow 0$ ). For a slow incoming flow, a rarefaction wave will be formed near the (absorbing) wall, propagating against the flow, until it reaches the source, where it interferes with

its action. The formation of a rarefaction wave was nicely demonstrated in Ref. [21]. Note that the "tip" of a rarefaction wave propagates relative to the upstream flow with the sound speed (e.g., [22]). Hence, for $\eta<1$, 
the problem of plasma creation becomes inseparable from the analysis of the downstream conditions and one is forced to consider the entire flow problem at once. In this sense, the parameter $\eta$ can be called a "connectivity parameter": if $\eta<1$, sound waves can connect the sheath region to the source whereas for $\eta>1$ this cannot not happen.

In the "global" problem that one has to consider at $\eta<1$, one is not free to assign a specific value to the flow velocity at the boundary; it emerges from the solution. It is quite possible, as we will see in Sec. 2.4, that the ions will be pre-accelerated in the source region to reach a supercritical flow. Ion pre-acceleration by the ambipolar electric field in the source region was considered in Ref. [23] for the case of the ions injected at a zero energy and in Ref. [24] for a source that injects ions with a distribution function of the form $\left|v_{z}\right| \exp \left(-\operatorname{const} \cdot v_{z}^{2}\right)$. In Ref. [25] it was shown that, for both solutions, the condition $\eta=1$ holds downstream from the source region. However, this conclusion can be questioned, because it was drawn for potentials approaching 0 from above, whereas the condition (2) has to be satisfied for $\Phi<0$. This issue will be further discussed in Sec. 2.3.

One can find the connectivity parameter in the case of a more general ion distribution function $f\left(v_{z}\right)$ (nonzero at $v_{z}>0$ ), by taking a moment of the Vlasov equation to evaluate $\partial n_{i} / \partial \Phi$ in Eq. (2). If the distribution function is normalized to unit density, the condition becomes

$$
\eta=-\frac{T}{M}\left[\int_{-0}^{\infty} \frac{d v_{z}}{v_{z}} \frac{\partial f}{\partial v_{z}}\right]^{-1}=\frac{T}{M}\left[\int_{-0}^{\infty} \frac{f d v_{z}}{v_{z}^{2}}\right]^{-1}>1
$$

We write the lower integration limit as -0 in ordr to include a possible jump in the distribution function at $\mathrm{v}_{z}=0$; if such a jump is present (as is the case for a half-Maxwellian), the integral diverges, making $\eta=0$. Note that in the solution of Ref. [24], even at a high initial ion temperature, the result is a critical flow. This is entirely related to the assumption regarding the distribution function: in the absence of an accelerating field it is a halfMaxwellian. In reality collisions render this situation impossible near the wall and guarantee convergence of the integral; see Sec. 2.5 below.

It is sometimes debated how one should define the "sheath entrance." Although a definition "a few Debye radii from the wall" seems to be adequate in the supercritical case, it may not be in the critical or sub-critical case. This is related to the coupling of sheath and source regions and will be discussed further in Sec. 2.3.

\subsection{More on the Bohm criterion as a "connectivity" condition}

To emphasize the role of the Bohm condition as the connectivity condition, we consider a problem where a sheath per se is not present at all. This is the situation where the cross-section of an initially uniform flux tube begins to expand beyond some point $z=z_{0}$ and then flares up into vacuum as shown in Fig. 2. This problem is relevant, in particular, to expandersof mirror devices and may be important for innovative designs of tokamak divertors. As the density in the flaring region will drop, a potential variation will be forced

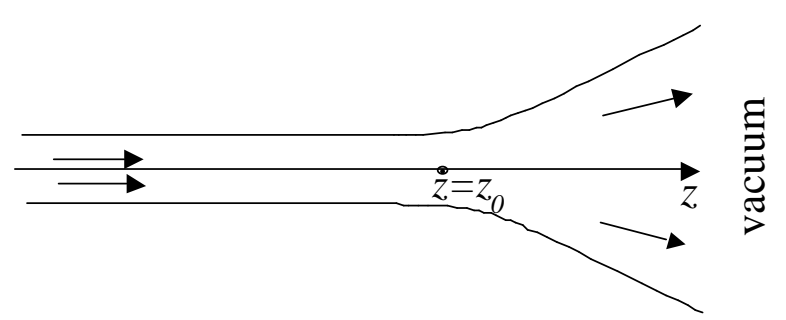

Fig. 2 Plasma flow in an expanding flux tube. The sources are situated to the right, outside the boundaries of the figure. A flow which is initially uniform expands into the flaring section, causing formation of an ambipolar potential. to occur there: $\partial \Phi / \partial z=\left(T_{e} / n e\right) \partial n / \partial z$.

The dependence of the potential on the expansion ratio $A(z)=\operatorname{Area}(z) / \operatorname{Area}(0)$ is determined from the quasineutrality constraint, which, for a mono-energetic flow, is $(1 / A)(1-\phi / \eta)^{-1 / 2}=\exp \phi$, where $\phi=e \Phi / T_{e}$. One easily finds that solution of this equation exists only for $\eta>1$, the same result as for flow into an absorbing wall. The result extends also to a more general distribution with $\eta$ given as in Eq. (3). What happens in the opposite case $\eta<1$ is the same as in the case of an absorbing wall: the plasma expansion to vacuum causes propagation of the rarefaction wave against the upstream flow and the source region becomes affected by the downstream conditions. 


\subsection{A "global" solution for a simple model.}

To illustrate the connectivity condition in the case of a sheath, we consider a model where a simple analytical solution exists for the global problem which uniformly treats both the sheath and source regions. We consider the following ion kinetic equation: $d f_{i} / d t=S(z, v)$, where $S$ represents the source term, and the derivative is taken along the ion trajectory in phase space. (We actually consider only a steady-state problem.) We assume that the source region is localized in the middle of the flux tube and is symmetric with respect to the midpoint $z=0$. The walls are situated at $z= \pm L$. At every point the source generates equal amounts of ions moving to left and to right with equal velocities $u$, i.e., $S(z, v)=q(z)[\delta(v-u)+\delta(v+u)]$. The function $q(z)$ is some smooth even function which decreases rapidly outside the source region (say, in a Gaussian fashion), so that there is a substantial source-free gap between the source region and the wall. The total number of ions generated by such a source and moving, say, to the right wall is $\int_{-\infty}^{+\infty} q(z) d z$. The results presented below can be easily extended to the case of ions injected symmetrically with a smooth velocity distribution function; an important constraint though is that there is some minimum ion velocity $u_{0}$ so that the source is zero at $v<u_{0}$.

We will study the regime of supercritical flow, $\eta>1$, and will trace the changes that occur when $\eta$ approaches unity. As we shall see, in the source region the potential variation remains small and there are no turning points for the ions, i.e., $-e \Phi(z)<M u^{2} / 2$. Taking the potential at the midpoint as zero, one can write the following equation for the ion density:

$$
n_{i}(z)=\frac{2}{u} \int_{0}^{\infty} \frac{q\left(z^{\prime}\right) d z^{\prime}}{\sqrt{1+(2 / \eta)\left[\phi\left(z^{\prime}\right)-\phi(z)\right]}}
$$

where the denominator does not have zeros in the source region. The electron density is given by equation $n_{e}=n_{e}(0) \exp \phi$. Note that we do not impose a quasineutrality constraint. If there were no absorbing wall, there would have been a very simple solution to the Poisson equation (2) in this area: just a uniform plasma, with $\Phi=0$ everywhere, and the density being strictly neutral and given by $n=n_{0} \equiv(2 / u) \int_{0}^{\infty} q(z) d z$ both inside and outside the source region. In order to match to the boundary condition on the wall, we need, however, to consider deviations from this solution. As will be shown, the potential is small beyond a few $\lambda_{D}$ from the wall, unless one comes too close to the critical condition $\eta=1$ (see below). This means that one can use the following expansions for the particle densities (up to linear terms in the potential): $n_{i}(z)=n_{i}(0)(1+\phi / \eta)$ with $n_{i}(0)=(2 / u) \int_{0}^{\infty} q(z)[1-\phi(z) / \eta] d z^{\prime}$ In the same approximation, $n_{e}(z)=n_{e}(0)(1+\phi)$. (Near the walls, where the potential becomes substantial, we would need the exact expressions for the densities.) The Poisson equation, therefore, is:

$$
\phi^{\prime \prime}=k^{2}[-\delta+\phi(\eta-1-\delta)]
$$

where $k^{2}=4 \pi n_{i}(0) e^{2} / T_{e}, \delta=1-n_{e}(0) / n_{i}(0)$ and we assumed that $|\eta-1| \ll 1$. The solution of this equation satisfying the boundary conditions $\phi(0)=0 ; \phi^{\prime}(0)=0$ is:

$$
\phi=\frac{\delta}{\eta-1-\delta}[1-\cosh (k z \sqrt{\eta-1-\delta})]
$$

The parameter $\delta$ has to be chosen from matching this solution to the solution in the immediate $(z-L \sim$ a few $\lambda_{D}$ ) vicinity of the wall, where $y$ becomes, say, 0.1 . Without getting into too much detail, we just note that, for $\eta-1>>1 / k^{2} L^{2} \sim \lambda_{D}^{2} / L^{2} \delta$ is exponentially small, $\ln (1 / \delta) \sim k L /(\eta-1)^{1 / 2}$. In this case, in agreement with the discussion of Secs. 2.1 and 2.2, the effect of the presence of the absorbing wall on the upstream flow and the source region is negligible. Conversely, if $\eta$ becomes close to $1, \eta-1<(k L)^{-2}$, the situation qualitatively changes. In this case the potential varies quadratically instead of exponentially over the bulk of the source region, such that the potential is of order unity rather than exponentially small. The potential near the plasma side of the sheath decreases (asymptotically) as $1 /(L-z)^{2}$ [4] (and not exponentially).

\subsection{Delta-function source.}

There is an even simpler case where an analytical solution can be built for both super- and sub-critical cases. Namely, we assume that the source region becomes infinitesimally thin, $S(z, v)=q_{0} \delta(z)[\delta(v-u)+\delta(v+u)]$. 
For this source one obtains the following equation for the dimensionless potential $\phi$ :

$$
\phi^{\prime \prime}=(1-\delta) \exp \phi-\left(1-\frac{2 \phi}{\eta}\right)^{-1 / 2}
$$

In this section, the coordinate $\mathrm{z}$ is normalized to $k^{-1}$. The boundary condition (1) has to be imposed at $z=k L \gg 1$. The other two boundary conditions are $\phi(0)=0 ; \phi^{\prime}(0)=0$. Equation (26) has a first integral, and can be presented in the following form allowing for the use of a simple mechanical analogy: $\phi^{\prime \prime}=F \equiv-\partial U / \partial \phi$ where

$$
U=(1-\delta)(1-\exp \phi)+\eta(1-\sqrt{1-2 \phi / \eta})
$$

If one identifies $\phi$ with a spatial coordinate of a particle in a mechanical system, and $z$ with time, one sees that Eq. (27) describes the motion of this object in the potential (4) (we have chosen an arbitrary constant in $U$ so as to make it zero at the origin).

We will consider here only the most intriguing subcritical case, assuming that $\eta$ is less than 1 by order one. We consider motion of a particle that starts at the origin (at $\phi=0$ ) and has a zero initial velocity, $\phi^{\prime}=0$, and falls to large negative values of $\phi$ determined by the boundary condition (1). One immediately sees that this is possible only for $\delta>0$, as otherwise the potential near zero would have looked as shown by dashed line in Fig. 3, and the "particle" would stay in the $\phi \geq 0$ region. The shape of the potential curve depends on $\delta$. In Fig. 3 the solid line depicts the curve that corresponds to the physical nature of the problem. It has a maximum which must lie slightly below the horizontal axis. The

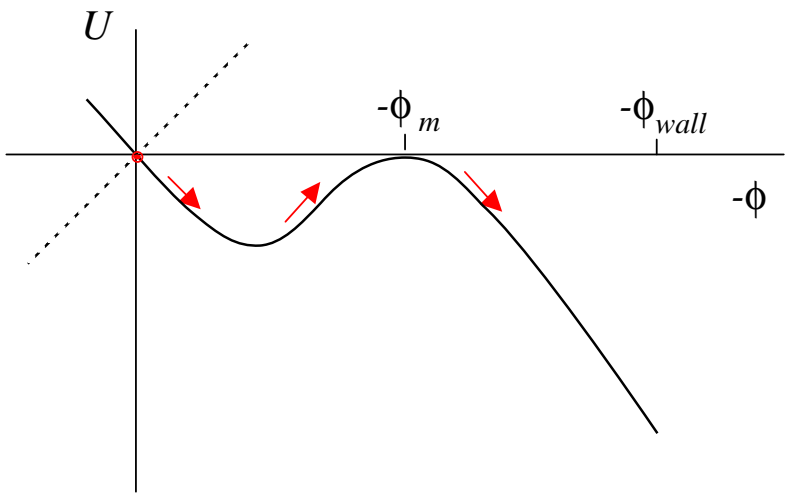

Fig. 3 Effective potential for the delta-function source problem. latter statement is related to the notion that the time that particle has to spend traveling to the end wall (1) must be very long. So, the particle must almost stop in the vicinity of the potential maximum and spend a very long timethere before starting to slide to the wall. In the real system this corresponds to a long zone of a slowly varying potential between the source and the Debye sheath. The point where this happens is found from the following two equations: $F\left(\phi_{m}\right)=0 ; U\left(\phi_{m}\right)=0$, which can be solved (numerically) for the parameters $\delta$ and $\phi_{m}$. From the results we can determine the normalized energy $m u^{2} / T_{e}=\eta+2 \phi_{m}$ with which ions approach the wall It is always supercritical, $\eta+2 \phi_{m}>1$. In particular if the source itself is strongly sub-critical $\eta \ll 1$, then $\phi_{m} \approx 1.26$.

In conclusion to sections 2.3 and 2.4: The condition (2) is sometimes considered as a boundary condition for the inner part of the problem. However, this condition by itself doesn't tell us very much, and in particular, our examples illustrate that it needn't be satisfied in its equality form for either supercritical or subcritical sources. One has to actually solve the problem in its entirety, bearing in mind that, for sources generating fast supercritical flows, the source region is independent of the conditions downstream, whereas the slow flows do connect the source region with the conditions downstream.

\subsection{Collisional flows}

Now we briefly discuss the collisional case, where the characteristic particle mean-free-path $\lambda_{\text {coll }}$ is much smaller than the distance between the walls and the length of the source region but still much larger than the Debye length.

A few men free paths from the wall, the ion distribution function is a shifted Maxwellian, with some average velocity (flow welocity) $u$. A qualitative formulation of the fact that the wall is perfectly absorbing is the identification of the wall with a hole, beyond which the flow is flaring out into the large empty space. Then, solving the inner part of the gas-dynamical problem immediately leads to an identification of the flow velocity at the wall with the sound velocity $c_{s}=\left\{\left[(5 / 3) T_{i}+T_{e}\right] / M\right\}^{1 / 2}$. This line of reasoning was suggested in Ref. [8]. 
A more rigorous approach would require a kinetic analysis of the several mean free paths nearest the wall. At the plasma side of the Debye sheath, there are no ions coming back to the plasma. On the other hand, in the hydrodynamic model, the shifted Maxwellian distribution has some number of particles moving in the reverse direction. However, their fraction $\epsilon$ is small:

$$
\epsilon=\pi^{-1 / 2} \int_{\left[(5 / 6)+\left(T_{e} / 2 T_{i}\right)\right]^{1 / 2}}^{\infty} e^{-x^{2}} d x \approx \frac{\exp \left[-(5 / 6)-\left(T_{e} / 2 T_{i}\right)\right]}{2\left\{\pi\left[(5 / 6)+\left(T_{e} / 2 T_{i}\right)\right]\right\}^{1 / 2}}
$$

Even at $T_{e}=0, \epsilon$ is quite small, $\sim 0.13$, suggesting that the solution of a complete kinetic problem wouldn't depart much from the results of a simple hydrodynamic treatment.

We also note that, for Coulomb collisions, we expect that the ion distribution function at the wall will have a smooth approach to $v_{z}=0$ (quadratic, $f \propto v_{z}^{2}$, or smoother), because the main contribution to the Coulomb collision integral evaluated near the $v_{z}=0$ point is proportional to $\partial^{2} f / \partial v_{z}^{2}$. A less-smooth transition (say linear in $v_{z}$ ) would yield an infinity in the kinetic equation. Together with the observation that there is no characteristic scale in the ion distribution function other than the ion thermal speed $v_{t i}$ (for $T_{e}$ not too much higher than $T_{i}$, as is the case in the tokamak SOL), one can conclude that the integral (3) should converge and be on the order of $1 / v_{t i}^{2}$.

As we have already mentioned, the ion temperature in a tokamak SOL often exceeds the electron temperature, sometimes substantially. Under these circumstances the plasma approaching the Debye sheath is supercritical. As we have seen above, this means that no pre-acceleration is required for connectivity and a monotonic sheath structure is assured. Thus we have both collisional and collisionless examples of plasmas for which the upstream flow conditions and connectivity set the flow speed to be finitely greater than critical. For the collisional case with an effective nozzle upstream from the wall, a sonic transition occurs at the nozzle and the flow at the wall can be finitely greater than the critical value. But in this case the connectivity criterion is set by the nozzle; conditions at the wall (other than that it be absorbing) are irrelelvant to the flow upstream of the nozzle.

\section{Oblique Incidence}

Magnetic-fusion edge plasmas are characterized by magnetic fields which intersect the end wall typically at an oblique angle; in the common case of a tokamak with a poloidal divertor (and also one with a toroidally symmetric limiter), the field lines are almost grazing; the angle of incidence for a divertor plasma is typically of order several degrees. This same situation can also occur in some gas discharge devices and in the vicinity of spacecraft.

As noted in Refs. $[6,16,17]$, the physics of the sheath-presheath transition is substantially different in the oblique case compared to normal incidence; in particular, a two-scale structure develops: a Debye sheath, with a scale length of the order of the Debye length, exists (except under special circumstances) immediately next to the end wall; beyond that, a "gyrosheath" inevitably forms, with a scale length of the order of the sound gyroradius $\rho_{s}=\left[\left(T_{e}+T_{i}\right) / m\right]^{1 / 2} / \omega_{c i}$, due to the effects of ion scrapeoff. As noted in Ref. [16], ion scrape-off in the gyrosheath leads to a significant density drop and hence a transverse electric field $\sim T_{e} / e \rho_{s}$ and significant ion orbit distortion, in effect guaranteeing that ions enter the Debye sheath with a normal velocity at least of order of the Bohm velocity. A typical ion orbit and the potential structure are sketched in Fig. 4.

For small angles of incidence, effects other than parallel flow play an important role in setting boundary conditions. In particular, cross-field flows due to drifts [7, 8, 9], collisions [13, 17] and radial transport can compete with parallel flow. In the treatments of $[13,17,18]$, the mechanism for these flows influencing boundary conditions is that they compete as sources of transport of mass to bounding surfaces. We shall argue below that the competition is really for flow perpendicular to the symmetry direction, and in particular that mass flow boundary conditions are insensitive to the orientation of the end plates (except for almost-perfectly grazing conditions). On the other hand the competing flows onto the plates plays a central role in current and energy boundary conditions.

\subsection{Mass flow}

We consider first the question of velocity boundary conditions at the magnetic presheath entrance. A variety of approaches have appeared in the literature: Chodura [6] examined the dispersion relation for Debye-scale perturbations of a fluid in a local slice of the quasineutral plasma at the presheath entrance, rejecting oscillatory 

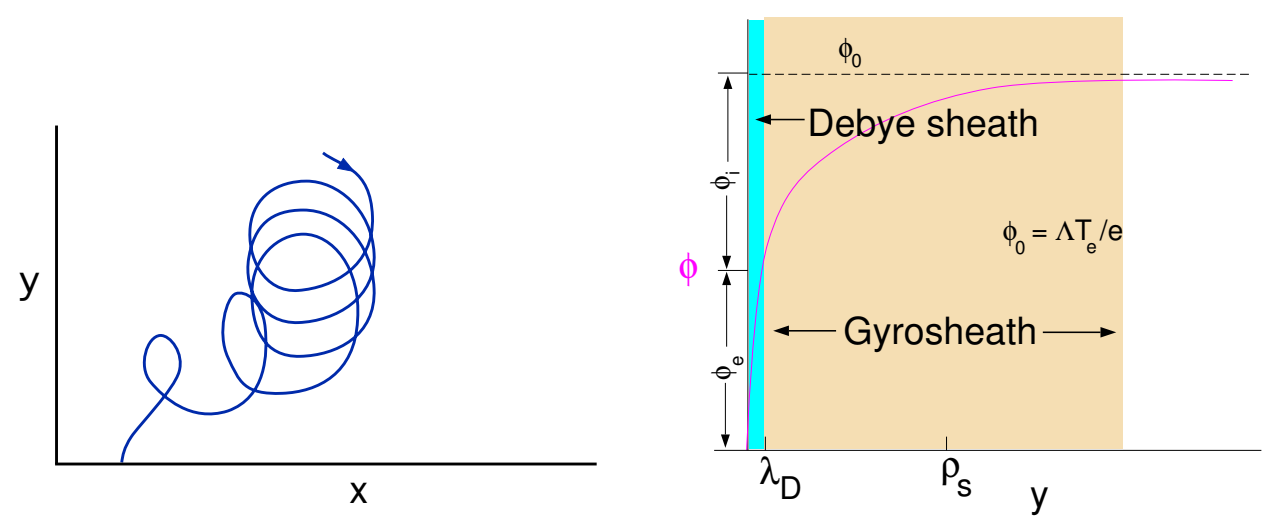

Fig. 4 Sketches of typical ion orbit and of potential variation in gyrosheath and Debye sheath regions.

solutions as incompatable with transition to the Debye sheath. The results were compared with nonlocal numerical simulations of kinetic and fluid models which suggest that $v_{\|} \gtrsim c_{s}$ is required to avoid potential oscillations in the gyrosheath . Classen and Gerhauser[10] invoke a criterion that the extension of the sheath solution should continue to decay monotonically across the gyrosheath and from this arrive at a criterion that $d n_{e} / d y \geq d n_{i} / d y$ at the gyrosheath entrance, then evaluate the criterion from a moment of Vlasov equation. Here $y$ denotes length normal to the wall. Riemann [13] and Tskhakaya and Kuhn [17] analyzed the allowed forms of quasineutral fluid solutions across the gyrosheath consistent with the Bohm velocity on the wall (Debye-sheath) side and an asymptotically constant solution on the plasma side. Cohen and Ryutov [8,9] invoked the analogy of a perfectly abosrbing boundary to flow through a nozzle.

These approaches all have some drawbacks: First, Refs. [6, 13, 17] all suffer from application of fluid equations on a distance scale where they cannot be valid (Debye and gyroradius scales, respectively). Within the gyrosheath, ion orbit distortion and scrapeoff implies that kinetic corrections to fluid equations must be significant $[16,15]$. This is directly a problem for the analyses $[13,17]$ which integrate fluid equations up to the Debye sheath edge. The dispersion-relation approach [6] is a local analysis which could be applied on the plasma side of the gyrosheath where the strong orbit distortion effects are absent; but the applicability of fluid theory for Debye-scale variations is still dubious. Also this analysis could equally well be applied to a source-free region far frorm the boundary but separated from the boundary by sources; the flow is sub-sonic, and so an oscillatory perturbation here is acceptable. The only reason for it being unacceptable near the gyrosheath must then be related to the inability to connect it to the Debye-sheath solution, but that connection must be made across the layer where strong orbit distortions render the fluid equations inadequate. The Claasen-Gerhauser approach [10] rests on three questionable assertions: that normal-to-sheath derivatives are larger than radial derivatives at the gyrosheath entrance (as opposed to well within the within the gyrosheath), and that the potential solution must monotonically decay all across the gyrosheath, and that this implies that $\partial^{3} \Phi / \partial y^{3}>0$ at the gyrosheath entrance. As noted in the previous section, the nozzle analogy has the limitation that the condition of no mass flow backward through the nozzle is not the same as no backward-going particles in the distribution function, though the distinction is likely significant only for $T_{e}<T_{i}$. Fortunately, as noted in Sec. 2, for a perfectly absorbing wall, the limiting velocity is just a function of the bulk-plasma solution, subject only to the constraint that that there be no flow from the sheath region back into the main plasma. That is, if we can define a test surface beyond which there are no sources and no nozzles (constriction of a flux tube), then, so long as there is no back flow through the control surface, conditions at the control surface cannot be affected by conditions beyond it. Hence any procedure that meets this criterion will do; our choice here is to use the nozzle analogy as it relies only on the fluid description in the bulk plasma.

There have been some attempts to introduce corrections to gyrosheath theory based on fluid analysis of phenomena argued to be important within the gyrosheath, such as variation of the $\mathbf{E} \times \mathbf{B}$ speed [18] and cross-field transport induced by collisions with neutrals $[13,17,18]$; such extensions must be viewed with suspicion because of the noted limitations of the fluid theory in that region. In the nozzle approach these phenomena also appear, as will be seen below, though only in the form of bulk-plasma drifts; the resulting predictions for the critical 
velocity differ from those from a fluid gyrosheath analysis. It should also be noted that the premise [18] of an electric field tangential to the end plate which smoothly drops to zero across the gyrosheath is incorrect; much of this drop occurs within the Debye sheath very close to the end plate.

We note that there have been a number of attempts to treat the gyrosheath problem kinetically via simulation techniques $[6,14,15,17,18]$. These approaches are valuable for the insights they provide, and have generally confirmed some basic results obtained in the fluid analysis (see the above references and the treatment below): that the ions enter the Debye sheath with a normal velocity satisfying the Bohm criterion, and that, in the absence of drifts, they approach the gyrosheath with a parallel velocity satisfying the Bohm criterion. They can also address a regime of high ion-neutral collisionality not yet accessable (for reasons which vary with the method) for the various analytic approaches described above; we shall discuss these results along with our discussion of the nozzle model results below. Some of the above authors have also performed numerical solutions of fluid equations across the gyrosheath for comparison purposes, but given the limitations of the fluid equations, we will not discuss these here. All of the numerical treatments involve some caveats and numerical uncertainties, and of course can provide only limited information on scaling in a multi-dimensional space of parameters.

We follow the treatment of Ref. [9], adding to it effects of neutral-particle collisions and explicitly calling out the role of turbulent radial transport. Considering only axisymmetric systems and taking into account that the $\mathbf{E} \times \mathbf{B}$ drift velocity is divergence-free, the continuity equation can be written in the form:

$$
n \frac{\partial v_{\|}}{\partial s}+\hat{v}_{\|} \frac{\partial n}{\partial s}+n \frac{\partial B v_{\nu, p} / B_{p}}{\partial s}+v_{E r} \frac{\partial n}{\partial r}=S_{n, e f f}+n v_{\|} \frac{\partial \ln B}{\partial s}
$$

where $v_{E r}$ and $v_{E p}$ are the "radial" (normal to flux surface) and poloidal $\mathbf{E} \times \mathbf{B}$ drift velocity components, $\hat{v}_{\|}=v_{\|}+\left(B / B_{p}\right)\left(v_{E p}+v_{\nu, p}\right), B_{p}$ is the poloidal magnetic field component, and $s$ denotes length along a field line. We consider $B_{p} / B$ small and so neglect the distinction between $B$ and the toroidal field $B_{T}$. We have introduced an effect of neutral particle collisions through the induced poloidal drift $v_{\nu, p}=\hat{\nu} c E_{p o l} / B\left(1+\hat{\nu}^{2}\right)$. Here $\hat{\nu}=\nu / \omega_{c i}$ and $\omega_{c i}$ is the ion cyclotron frequency. Having introduced this drift, we note that we can safely neglect it, as $v_{\nu, p} / v_{E p} \hat{\nu} L_{r} / L_{p} \ll 1$, where $L_{r}$ and $L_{p}$ are the local radial and poloidal scale lengths. The corresponding radial component is not negligible and is included in the effective source $S_{n, e f f}$, which also includes turbulent radial transport terms: $S_{n, \text { eff }}=S_{n}-(\partial / \partial r)\left(n v_{\nu, r}+\Gamma_{\text {turb }}\right)$

Similarly the parallel momentum equation (summed over electrons and ions) is:

$$
m n \hat{v}_{\|} \frac{\partial v_{\|}}{\partial s}+m n v_{E r} \frac{\partial v_{\|}}{\partial r}=-\frac{\partial p}{\partial s}+S_{v, e f f}
$$

where $m$ is the ion mass and $S_{v, \text { eff }}$ is the effective momentum source $-m v_{\|} S_{n}-\nu_{c x} n v_{\|}$plus any physical momentum-density source and corrections for neutral-particle driven and turbulent radial momentum flux, as above; here $\nu_{c x}$ is is the charge-exchange rate on cold neutrals. The $\nu_{c x}$ term represents a second effect of neutral-particle collisions.

We note that $v_{E r}$ in the above equations is proportional to the poloidal electric field and hence, because of axisymmetry, $v_{E r} \approx-\left(c / B_{p}\right) \sigma_{t} \partial \Phi / \partial s$, where $\sigma_{t}=\operatorname{sign}\left(B_{t} / B\right)$ and where we consider the radial, poloidal and toroidal directions to form a right-handed coordinate system and take the path length $s$ along the magnetic field to increase in the direction of $\mathbf{B}$. Using the electron momentum equation, this becomes

$$
v_{E r}=-\frac{c \sigma_{t}}{e B_{p}}\left(1.71 \frac{\partial P_{e}}{\partial s}-0.71 T \frac{\partial n}{\partial s}\right)
$$

We can consider any of several models in which $\partial p / \partial s$ is proportional to $T_{e} \partial n / \partial s$ plus, possibly, a sink term with no parallel derivatives of plasma quantities, so that

$$
\partial p_{e} / \partial s=\gamma_{e f f, e} T_{e} \partial n / \partial s+S_{p s}
$$

where the constant $\gamma_{e f f, e}$ and the effective source $S_{p s}$ depend on the electron-pressure closure model. For isothermal electrons, $\gamma_{e f f, e}=1$ and $S_{p s}=0$. For adiabatic electrons with ratio of specific heats $\gamma, \gamma_{e f f, e}=\gamma$ and $S_{p s}=0$. We also consider a flux-limited model in which the divergence of the parallel electron heat flux is equal to an explicit sink term $\pm S_{h}$ resulting from radial transport; here + (-) sign refers to the high- $s$ (low- $s$ ) ends 
of the flux tube and $S_{h}$ is taken as a negative quantity when the radial transport represents a net loss of heat from the flux tube. In this case we have $\gamma_{e f f, e}=1 / 3$ and $S_{p s}=(2 / 3)\left[\left( \pm / c_{s e}\right)-n T_{e} \partial \log B / \partial s\right]$. Finally, we note that $P=P_{e}+P_{i}$, and evaluate $\partial P_{i} / \partial s$ assuming the ions to be adiabatic (with an adiabatic index $\gamma_{i}$ which may be different from $\gamma_{e f f, e}$. Substituting Eq. (9), the corresponding relation for ions, and Eq. (8) in Eqs. (6) and (7) and eliminating $\partial n / \partial s$ between the two equations yields an equation for $\partial v_{\|} / \partial s$,

$$
\hat{v}_{\|}\left(1-\frac{c_{s, e f f}^{2}}{\hat{v}_{\|} v_{\|}^{\dagger}}\right) \frac{\partial v_{\|}}{\partial s}=-n v_{\|} \frac{\partial \ln B}{\partial s}+\frac{\hat{S}_{v}}{m n}-\frac{\hat{S}_{n} c_{s, \text { eff }}^{2}}{n v_{\|}^{\dagger}}
$$

where $\left.v_{\|}^{\dagger}=\hat{v}_{\|}+\hat{\gamma} \sigma_{t} c_{s e} \rho_{s e, p} / L_{n}, c_{s, e f f}^{2}=\left(\gamma_{e f f, e} T_{e}+\gamma_{i} T_{i}\right) / m+\hat{\gamma} c_{s e} \rho_{s e, p} \sigma_{t} / v_{\|} L_{v}\right), \hat{\gamma}=1.71 \gamma_{e f f, e}-0.71$, $c_{s e}=\left(T_{e} / m\right)^{1 / 2}, \rho_{s e, p}=c_{s e} / \omega_{c i, p}, \omega_{c i, p}=e B_{p} / m c, L_{n} \equiv-n /(d n / d r)$, and $L_{v}=-v_{\|} /\left(d v_{\|} / d r\right)$, and where $\hat{S}_{n}$ and $\hat{S}_{v}$ are the effective source terms that appear in the continuity and momentum equations after all of the above substitution; they are given by $\hat{S}_{n}=S_{n, \text { eff }}+1.71 S_{h} \sigma_{t} / m \omega_{c i, p} L_{n}$ and $\hat{S}_{v}=S_{v, \text { eff }}+S_{p s}(1+$ $\left.1.71 v_{\|} / \omega_{c i, p} L_{v}\right)$.

We use Eq. (10) to model a situation with absorbing walls by noting that absorbing walls are (almost) equivalent to a situation where the wall is replaced by an infinite source-free extension of the flux tubes in which the flux tubes continuously expand ( $B$ decreases) as does the parallel velocity. From Eq. (10), the coefficient of $\partial v_{\|} / \partial s$ must thus be positive in this region, and thus positive or zero on the boundary. This gives us our generalized "Bohm" criterion; for the isorthermal or adiabtic electron pressure models and no drifts, it gives just the criterion $v_{\|} \geq c_{s}$ derived by Chodura for the parallel velocity at the entrance to the gyrosheath. When we retain the poloidal drifts but drop the (small) radial $\mathbf{E} \times \mathbf{B}$ drift, we just replace $v_{\|}$by $v_{\|}+B / B_{p}$ times the poloidal drift.

Note, the right-hand side of Eq. (10) is positive beyond the wall but is necessarily negative somewhere in the interior of the flux tube where the particle flux is appreciable and $v_{\|}$is small; likewise the left-hand side of Eq. (10) is negative there. The two sides of the equation must pass through zero at the same physical point, which may be at the boundary or in the interior, depending on the distribution of sources/sinks and the field strength. The generalized Bohm criterion as an equality should be satisfied at this point. We note that, for a near-detached divertor situation where the temperature drops appreciably in the divertor leg, one may well have a situation where the net source term changes from positive (ionization dominating) to negative (recombination and/or loss processes dominatng); the effective nozzle (and hence a supersonic transition) may occur in the transition between these regions.

For the simple case of a uniform radial electric field (and neglecting the poloidal electric field), the above results can be derived more simply by transforming to a toroidally rotating frame in which the electric field vanishes. In the rotating frame, the sources and the effective nozzle are stationary, but the source parallel velocity distribution is shifted by $v_{E p} B / B_{p}$. Since there is no drift-producing electric field, the parallel flow in this frame must be sonic at the nozzle, and so satisfies the conventional Bohm criterion at the gyrosheath entrance. Transforming back to the lab frame, the shifted Bohm criterion derived above is obtained.

It should be noted that the criterion obtained above is to be used with bulk plasma parameters, not parmeters inside the gyrosheath; the influence of the absorbing wall, including the gyrosheath, has been absorbed into the fictitious extension beyond the wall. Thus conceptually we are determining conditions at the boundary of a domain which does not include the gyrosheath. Thus the various drifts (including that induced by neutral collisions) should be evaluated in the bulk region.

We note that the drifts act in opposite senses relative to the parallel loss at opposite ends of a flux tube; hence the drifts induce asymmetries in the density (as well as current and heat) end loss rates and thus contribute to density and temperature asymmetries. As pointed out in Ref. (??) there are subtleties here; when account is taken of the natural variation of temperature and density along a field line, the effect of drifts on the density and temperatue asymmetries can be opposite to that which one would naively expect based on the direction of the drift flow.

The shift in the critical velocity due to the $\mathbf{E} \times \mathbf{B}$ drift generally agrees with those obtained by other studies $([11,12,10,7,18])$ (though Refs. [18] and [10] claim corrections associated with electric-field and (in the latter case) $v_{\|}$gradients, which we question because of the validity concerns raised above). We comment that, while the $\mathbf{E} \times \mathbf{B}$ and collision-induced drifts enter the velocity boundary condition, the diamagnetic drift does not. 
This is because the diamagnetic drift does not transport anything. In this regard our result differs from the earlier references ([11, 12]) but agrees with [7].

Simulations with neutral-collision-induced transport $([14,15,17,18])$ indicate that the collisions influence the entrance velocity, though there is no consensus as to the direction (not surprising, since, as pointed out above and in Ref. [15], there are competing effects). In constrast, in the nozzle analysis this transport enters only through the effective sources; it would be expected to contribute only to where the sonic transition occurs. A possible resolution is that the effect may become important only when the sources become sufficiently localized near gyrosheath, so that (for example) the neutral momentum source competes with $\mathbf{v} \times \mathbf{B}$. Then we may not be able to identify an effective nozzle without explicitly considering the gyrosheath region.

We remark about the $\left(B / B_{p}\right)$ factors that appear in $\hat{v}_{\|}$and thus enter the corrections to the sound speed in the critical velocity. The origin of this factor is that it is the sine of the angle between the magnetic field and the symmetry (toroidal) direction. It is only coincidentally equal to the sine of the angle between the magnetic field andthe endplate, or the cosine of the angle between the in-flux-surface drift velocity and the end plate. (Other components of the drift velocity (for example, the radial drift resulting from collisions) enter the effective source and so can affect where the critical velocity is achieved.) In particular if the end plate is slightly tilted toroidally, this tilt can signficantly alter the angle between field lines and the plate, but does not change the critical flow velocity so long as axisymmetry in the main scrape-off layer plasma is preserved. (And for a plate with varying toroidal tilt, it can be shown that the departure from axisymmetry induced from the toroidal variation in the potential discussed in Sec. 4 below leads to modifications of the effective source and not a change in the critical velocity). The cricitcal velocity is also insensitive to a radial tilt, at least until field lines are so nearly parallel to the wall that a sheath fails to form. This puts our results at odds with those from treatments that analyze fluid equations within the gyrosheath $([13,17,18])$, which predict that the critical mass flow velocity at the entrance to the gyrosheath should depend on the component of the drift velocity normal to the surface. But, as noted above, a fluid treatment within the gyrosheath is of questionable utility.

Finally we raise a point about the necessity of a monotonic sheath. At least two references $([10,18])$ raise possible corrections from radial gradients within the gyrosheath. We have pointed out validity concerns in the treatments. But suppose that these turn out to be correct requirements for a stationary, monotonic sheath, and that, for some configuration,. they lead to a requirement that the gyrosheath entrance velocity be finitely bigger than the (drift-corrected) sound speed at that location. If the flow speed is already supersonic but less than the above requirement, as a result of satisfying a nozzle constraint in the bulk plasma, there is no way that information from the gyrosheath can propagate to affect conditions up stream. The sheath would then have to adjust to the upstream flow conditions (by becoming non-monotonic and/or non-stationary), not the other way around.

\subsection{Current boundary conditions}

We consider here the current-voltage characteristics of the sheath, which set the boundary conditions for the current. These results were derived in Refs. [7, 9, 26]. For purposes of this discussion, by "sheath" we mean the combined Debye sheath and gyrosheath regions. Because of the oblique incidence, drifts as well as parallel currents enter. Unless the angle of incidence is very small (for $\theta<\left(m_{e} / m_{i}\right)^{1 / 2}$, cross-field ion orbit loss competes with parallel flow so that a traditional Debye sheath doesn't form), any ions entering the sheath region are swept to the wall by the sheath electric fields, and the ion contributon to the normal current, measured on the ion side of the sheath, is just

$$
j_{n i}^{(p)}=\alpha n e v_{\|}+\hat{\alpha}\left(j_{E p}+j_{d p i}\right)
$$

where $v_{\| i}$ is the ion parallel velocity at the gyrosheath entrance (see preceding sub-section), $j_{E p}$ and $j_{d p i}$ are the poloidal components of the ion $\mathbf{E} \times \mathbf{B}$ and diamagnetic currents, respectively: $j_{E p}=($ cne $/ B) \partial \Phi / \partial r$, $j_{d p i}=(c / B) \partial p_{i} / \partial r, \alpha$ is the sine of the angle between the field line and the bounding surface (divertor plate, etc.), and $\hat{\alpha}$ is cosine of the same angle. If the bounding surface is toroidally symmetric, then $\alpha$ is just $B_{p} / B$. The ion contribution on the wall side of the sheath is different, as currents flow parallel to the wall in the sheath; in fact the ion diamagnetic current closes in this region.

While drifts can compete with parallel flow for the ions for the $\alpha$ values of interest, they cannot compete with the thermal speed of electrons. The electron loss to the wall is impeded by the sheath potential, so that the current 
at the wall is just

$$
j_{n e}=\alpha j_{e 0}
$$

where $j_{e 0}$ is the parallel electron current that would escape over the same height sheath for normal incidence; for Maxwellian electrons, $j_{e 0} \approx-e n v_{t e} \exp \left(-e \Phi_{\text {sheath }} / T_{e}\right) /(2 \pi)^{1 / 2}$. Here $\Phi_{\text {sheath }}$ is the potential difference between the interior of the plasma and the wall. The normal current on the plasma side of the sheath is also given by Eq. (12) as the net electron current in the sheath is zero; the electric drift develops a piece which just cancels the sheath diamagnetic current. cancel [26]. This simple result hides some complications: the cancellation is incomplete for anisotropic electron distributions, so the current on the plasma side differs from Eq. (12); and also, the electric-drift component of the sheath current is closed by a parallel current from the wall, so that the parallel current in the bulk plasma is shifted from $j_{e 0}$ by just $1 / \alpha$ times the bulk $\mathbf{E} \times \mathbf{B}$ and diamagnetic currents, rendering the normal component given by Eq. (12).

Adding together Eqs. (11) and (12) gives the total current to the wall and constitutes the current boundary condition.

The drift currents are approximately divergence-free. Hence we can set the current entering one end of a flux tube equal to that leaving the other, plus any corrections from any non-neutrality of the particle source (including radial transport). This relation provides an equation for the electrostatic potential on the flux tube in terms of the plasma densities, temperatures, wall potentials, and drifts at each end of the flux tube. In general, one must solve the plasma equations in the interior of the flux tube to relate the plasma parameters at opposite ends. But in a simple situation of uniform temperature and density along the flux tube (a low-density limit), equal areas and drift velocities at both ends of a flux tube (applicable for an up-down symmetric double-null divertor configuration), and negligible source contribution, we directly obtain:

$$
\Phi-\Phi_{1 w}=\frac{T_{e}}{e} \log \frac{\left\{1+\exp \left[e\left(\Phi_{2 w}-\Phi_{1 w}\right) / T_{e}\right]\right\} v_{e}}{2 c_{s}+v_{D}\left(1 / \alpha_{2}-1 / \alpha_{1}\right)}
$$

where "1" and "2" refer to conditions at opposite ends of the flux tube; $\Phi_{j w}$ is the potential of the wall at end $j$ (which could differ from ground potential due to an applied bias potential or resistance in the external circuit), $v_{D}$ is the sum of the ion $\mathbf{E} \times \mathbf{B}$ and diamagnetic drift velocities, and $v_{e} \equiv\left(T_{e} / 2 \pi m_{e}\right)^{1 / 2}$.

\subsection{Temperature boundary conditions}

The temperature boundary conditions are provided from an analysis of the heat flux to the divertor plates; this is analagous to that done for the current. The heat flux normally incident on the wall is, generally, $q_{n}=\Gamma_{n}\left[W_{i}+\right.$ $\left.e\left(\Phi_{0}-\Phi_{w}\right)+2 T_{e}\right]$ where $W_{i}$ is the ion energy lost per escaping ion, $\Gamma_{n}$ is the particle flux along the field line, and $\Phi_{0}$ and $\Phi_{w}$ are, respectively, the plasma and wall potentials. There is an obvious division between the portions attributable to electrons and ions. For a collisional plasma, we have[30] $\Gamma_{n} W_{i}=(5 / 2) n T_{i} v_{\| i} \mathbf{b}+(3 / 2) n T_{i} v_{\mathbf{E} \times \mathbf{B}}$ (Stringer, PPCF '91)

For the particular case of normal incidence and uniform temperature and density, where one end of a flux tube is biased to a potential $\Phi_{b}$ and the other end grounded, we can express the heat fluxes to the each plate in terms of the (common) flux $q^{*}$ in the case of no bias, to obtain:

$$
q_{\text {bias }}-q^{*}=n u T_{e}(\ln \Psi-\phi+A \chi), \quad q_{\text {ground }}-q^{*}=n u T_{e}(\ln \Psi-\chi)
$$

where $\Psi \equiv\left(A+e^{\phi}\right) /(A+1), \chi \equiv 2\left(e^{\phi}-1\right) /\left(A+e^{\phi}\right)$, and $\phi \equiv e \Phi_{b} / T_{e}$. For small positive bias, the heat flux to the biased (grounded) end increases (decreases), while for $\phi \gtrsim 3$, the heat flux to both increases. The latter may be somewhat surprising, since there is still a strong drift away from one of the ends.

In discussing the mass flow conditions, one of the pressure closure relations discussed was that for fluxlimited electron heat conductivity. The conditions for flux limiting can be met in tokamak scrape-off layers, because the main contribution to the electron heat flux comes from the electrons with energies a few times the thermal energy and having, therefore, a much larger (50-100 times) mean free path than the thermal particles. The flux-limit model is a local approximation to a situation that is essentially non-local, and the tail of the electron distribution function can be appreciably distorted from Maxwellian. This, generally speaking, changes both the current-voltage characterisitics and the expression for the electron heat flux compared to the fully collisional case. This, however, has almost no influence on the gyrosheath structure, which is determined by the bulk electron distribution, which remains a local Maxwellian. 


\section{Consequences of Toroidal Asymmetry}

The potential that follows from the current boundary conditions [Eq. (13) or a generalization] depends on the angle between field lines and bounding surfaces, the density and temperature, and whatever constitutive relationships govern the connection between the wall potentials at opposite ends of a field line. Examples of the latter are the resistance of an external circuit connecting the two ends or a bias voltage applied between the two ends. Hence, by deliberately introducing variations in applied bias, external circuit resistance, the orientation of the bounding surface, or external gas feed, one can manipulate the plasma potential $[19,20]$. By introducing such variations asymmetrically in the toroidal direction (for example, by toroidally rippling the divertor plate, or by introducing toroidally segmented divertor plates with varying bias voltage or resistance to ground), one can induce toroidal variations in the plasma potential and hence a toroidally varying radial drift. If the toroidal asymmetry is large enough, the resulting convection may give rise to hydrodynamic instability (Kelvin-Helmholtz), creating turbulent flows which could be used to broaden the scrape-off layer and hence alleviate divertor heat-load problems.

The task could be made even easier [19] by introducing both toroidal and radial variations. One can then create a pattern of convective cells stirring the plasma.

These ideas make sense so long as the plasma potential is substantially influenced by contact with end walls; this would not be the case for an electrically detached divertor or if transport of all plasma quantities, including current, is dominated by radial convection (as one might imagine in the case of "blob" transport [27]). The effect would be present but weak for a high-recycling, high density SOL where the temperature drops substantially along field lines. But it offers the possibility of deliberately broadening the SOL for a moderate recycling case. Furthermore by introducing boundary perturbations of sufficiently high toroidal wavenumber, there is the possibility [20] that the plasma perturbations will be confined to the divertor leg, being washed out by finite gyroradius effects as they pass the vicinity of the separatrix x point.

These ideas are currently being tested in the MAST spherical torus, and the initial results are very encouraging [28]. MAST has a set of twelve radially oriented ribs at the bottom of the vacuum chamber, equally spaced in toroidal angle. In the experiment, every second rib is biased relative to ground; the remaining ribs are at ground potential. As the bias potential is increased, the stripe of heat flux on the biased ribs shifts as one would expect from the induced toroidal electric field pattern, and at the highest postive bias potentials, the stripes are appreciably broadened, as one may expect from simple estimates for onset of shear-flow-driven turbulence. The total power flow to the biased and unbiased ribs is asymmetric increased or decreased, respectively, as predicted by Eq. (14). Little effect is observed for negative bias. All of these results are as one would expect from simple theoretical considerations [20].

\section{Time-dependent boundary conditions}

The current boundary conditions of Sec. $3 \mathrm{~b}$ are derived under the assumption of a plasma steady state, and apply also to low-frequency instabilities. However, the gyrosheath, being of finite extent, takes a finite time to respond to changes in plasma conditions, and this gives rise to modifications in the current-voltage characteristics that become signficant at sufficiently high frequency. We summarize here the sem-quantitative derivation of boundary conditions given in Ref. [29].

We consider wavelengths long compared to a gyroradius and frequencies less than the cyclotron frequency. When time variations are taken into account, the ion current approaching the wall is unaltered from that of Eq. (11), as all ions approaching the gyrosheath fall through it, whether it is moving or not. For the electrons, the situation is different. The electrons transit the gyrosheath in a time much shorter than the wave frequency; hence the sheath is quasi-static, and the electron current at the wall is unaltered from Eq. (12). However, the current on the plasma side of the gyrosheath is now different, as the difference between it and the current at the wall must adjust to balance the change of the number of ions in the (quasi-neutral) gyrosheath. Hence the total current entering the gyrosheath from the plasma is

$$
j_{n}^{p}=e n\left[\alpha v_{e} \exp \left(-e \Phi / T_{e}\right)-\alpha v_{\| i}-v_{D}\right]+e \frac{d N}{d t}
$$


where $\Phi$ is the potential difference between the plasma and the (nearby) wall, and $v_{D}$ is the total ion drift velocity (electric plus diamagnetic) normal to the wall.

Equation (15) applies even to nonlinear problems, so long as they do not violate the assumption that all incident ions reach the wall. Ref. [29] goes on to estimate $d N / d t$ for small-amplitude (linear) perturbations. The largest effect (in the frequency range $\omega>\alpha \omega_{c i}$ where the time-dependent effects become significant) is that due to temperature fluctuations $\delta T_{e}$; as the temperature rises, so does the electric field normal to the wall in the gyrosheath, which produces a more effective acceleration and scrape-off of ions in that region. Hence

$$
\frac{d N}{d t} \sim-A\left(n \rho_{i} / T_{e}\right) \delta T_{e}-i \omega A n \rho_{i} \delta T_{e} / T_{e}
$$

where $A$ is a constant of order unity, and where the second form applies for perturbations of the form $\exp (-i \omega t)$. Comparing the linearization of the first term in Eq. (15) with Eq. (16), and using the equilibrium relation $v_{e} \exp \left(-e \Phi / T_{e}\right) \sim c_{s}$, we see that the time-dependent correction is term comparable to the others under the condition $\omega A \rho_{i} \delta T / T \sim n \alpha c_{s} e \delta \Phi / T$, or, for $\delta T \sim e \delta p h i$, this becomes $\omega \sim \alpha \omega_{c i}$.

\section{Summary and Discussion}

We have presented "boundary" conditions for the mass flow, current, and heat flux for use in fluid descriptions of a scrape-off layer plasma. We put quotes around "boundary", since the mass flow (Bohm criterion) condition is not really a boundary condition - it is a connectivity constraint which, in its equality form, is satisfied at a point which need not be at the field line end. In particular, for a collisional plasma, it occurs at an effective nozzle governed by the distribution of effective sources and the magnetic-field variation. The ion flow velocity at the actual boundary is then an output of the calculation. On the other hand, the current and heat flux boundary conditions really are boundary conditions (but depend on the ion flow velocity at the boundary).

Our one-dimensional analysis of collisionless plasmas illustrates a number of important points about the nature of this "connectivity constraint". First, the same criterion, and the same interior potential structure is obtained whether the field line is terminated by a wall with a Debye sheath, or by an open-ended region of expanding magnetic flux. Second, the nature of the solution is different for sub-critical and super-critical injection. For sub-critical injection, a rarefaction wave can propagate upstream to the source region and trigger production of a potential drop which provides pre-acceleration. But, contrary to what one might expect, the potential drop is not necessarily just that needed to excelerate to the critical velocity required for a monotonic sheath (our delta-function source solution always accelerates to a super-critical velocity for sub-critical injection). For supersonic injection, a rarefaction wave cannot propagate upstream; no pre-acceleration is needed; and in fact, for a symmetric source and a constant-cross-section flux tube, a constant-density solution is obtained up to near the entrance of the end region (sheath or flaring flux tube). We note that it is not hard for the effective source of a tokamak to be super-critical: this will tend to occur in low-recycling devices where the main particle source is radial transport, in which case $T_{i}$ tends to be larger than $T_{e}$ in the scrape-off layer.

For a collisional plasma, kinetic analysis, including collisions, of the region within a few mean free paths of the wall, is required to quantitatively evaluate the Bohm criterion and to assess the flow speed at the wall. This is because of the sensitivity of the Bohm criterion to the distribution function at low normal velocity on the one hand, and tendency of the Fokker-Planck collision operator to smooth the distribution function on the other. There may be an effective nozzle upstream from the wall, and in this case hydrodynamics dictates a supersonic transition at the nozzle. Then, once again, the detailed analysis of the sheath region has no influence on the solution upstream of the nozzle. If there is no upstream nozzle, then the nozzle analogy whereby the wall is replaced by a source-free expansion region beyond it, should provide a good approximation to the results of the detailed kinetic analysis.

For oblique incidence of field lines on walls, a two-scale potential structure (Debye-length and gyroradius scales) develops. The "gyrosheath" is inherently kinetic in nature due to the effects of ion acceleration and scrapeoff. This makes suspect analyses that rely on fluid equations applied to the gyrosheath. The "nozzle" approach surveyed here relies only on the applicability of fluid equations in the bulk plasma (in the general vicinity of the boundaries).

From the nozzle analysis we find, in general accord with other approaches, that there is a generalized "Bohm criterion" for oblique incidence: the parallel velocity shifted by the "unprojected" poloidal $\mathbf{E} \times \mathbf{B}$ velocity, 
$v_{E p} B / B_{p}$, must equal the sound speed at an effective nozzle point, which (as with normal incidence) can be at the gyrosheath entrance or further upstream. The diamagnetic drift does not enter this criterion (but does enter the current boundary condition). There are however corrections from the radial $\mathbf{E} \times \mathbf{B}$ velocity. The analysis can fail for sufficiently high ion-neutral collisionality that the effective source significantly overlaps with the gyrosheath.

We note that drifts compete with parallel flows when $c_{s} B / B_{p} \sim v_{\mathbf{E} \times \mathbf{B}}$. But in same limit, radial displacement of ions in gyrosheath becomes of order of the scrape-off layer width. The consequences when this limit is exceeded remain to be explored.

We observe that it is conceivable that the requirement for a monotonic, stationary sheath could under some circumstances lead to a flow velocity constraint that is finitely higher than that of generalized sonic flow through the effective nozzle. In this case the sheath must respond to the upstream flow, not vice versa, by becoming non-monotonic and/or non-stationary.

We note that the flow constraint depends (significantly) on the angle between $\mathbf{B}$ and the symmetry direction, while the current and heat-flux expressions (which are true boundary conditions) depend on the angle of incidence of $\mathbf{B}$ and the end wall. These will not be the same in the case of toroidally rippled divertor plates.

The dependence of the current boundary conditions, and hence the plasma voltage, on controllable parameters of the bounding surface leads to the possibility of inducing radial convection to broaden the scrape-off layer plasma through approaches such as toroidally asymmetric biasing or external resistivity, or varying wall materials, and toroidally rippled divertor surfaces. Preliminary tests on the MAST spherical tokamak are encouraging.

Acknowledgements Work performed under the auspices of the U.S. Department of Energy at the University of California Lawrence Livermore National Laboratory under contract W-7405-Eng.-48.

\section{References}

[1] I. Lagmuir, Phys. Rev. 33, 954 (1929).

[2] L. Tonks and I. Langmuir, Phys. Rev. bf 34, 876 (1929).

[3] D. Bohm, in The Characteristics of Electrical Discharges in Magnetic Fields, edited by A. Guthry and R. K. Wakerling (McGraw-Hill, New York, 1949), p. 77.

[4] K.-U. Riemann J. Phys. D 24, 493 (1991).

[5] P. C. Stangeby, "The plasma boundary of magnetic fusion devices." Institute of Physics Publishing, Bristol and Philadelphia, 2000.

[6] R. Chodura, Phys. Fluids 25, 16281982.

[7] A. V. Chankin and P. C. Stangeby, Plasma Phys. Controlled Fusion 36, 1485 (1994).

[8] R. H. Cohen and D. D. Ryutov, Comments Plasma Phys. Controlled Fusion 16, 255 (1995).

[9] R. H. Cohen and D. D. Ryutov, Phys. Plasmas 6, 1995 (1999).

[10] H. A. Claassen and H. Gerhauser, Contrib. Plas. Phys. 36, 361 (1996).

[11] D. A. Knoll and A. K. Prinja, J. Nucl. Materials 176-177, 562 (1990).

[12] H. Gerhauser and H. A. Claassen, J. Nucl. Materials 176-177, 721 (1990).

[13] K.-U. Riemann, Phys. Plasmas 1, 552 (1994)

[14] Th. Daube, K.-U. Riemann and H. Schmitz, Phys. Plasmas 5, 117 (1998).

[15] Th. Daube and K.-U. Riemann, Phys. Plasmas 6, 2409 (1999).

[16] R. H. Cohen and D. D. Ryutov, Phys. Plasmas 5, 808 (1998).

[17] D. Tskhakaya and S. Kuhn, J. Nucl. Materials 313-316, 1119 (2003).

[18] D. Tskhakaya and S. Kuhn, Contrib. Plas. Phys. 42, 302 (2002).

[19] R. H. Cohen and D. D. Ryutov, Nuc. Fusion 37, 621 (1997).

[20] R. H. Cohen and D. D. Ryutov, Contrib. Plas. Phys 42, 296 (2002).

[21] J.W. Cipolla, M.B. Silevitch, J. Plasma Phys. 25, 373 (1981).

[22] L.D. Landau, E.M..Lifshitz. 1987 "Fluid Mechanics" (Pergamon Press, New York)

[23] E. S. Harrison and W. B. Thompson, Proc. Phys. Soc. 74, 145 (1959).

[24] G. A. Emmert, R. M. Wieland, A. T. Mense, J. N. Davidson, Phys. Fluids 23, 803 (1980).

[25] R.C. Bissel, Phys. Fluids 30, 2264 (1987).

[26] R. H. Cohen and D. D. Ryutov, Phys. Plasmas 2, 2011 (1995).

[27] D. A. D'Ippolito, Contrib. Plas. Phys, this issue (2004).

[28] G.F.Counsell, J-W.Ahn, R.H.Cohen, A.Kirk, P.Helander,, R.Martin1, D.D.Ryutov, A.Tabasso, H.R.Wilson, Y.Yang, "Exhaust, ELM and Halo Physics using the MAST tokamak", in Fusion Energy 2002 (Proc. 19th Int. Conf. Lyon, 2002) (Vienna: IAEA) CD-ROM file exd1_2.pdf, http://www.iaea.org/programmes/ripc/physics/fec2002/html/fec2002.html/

[29] R. H. Cohen and D. D. Ryutov, Plasma Phys. Reports 23, 805 (1997) [Fizika Plazmy 23, 872 (1997)].

[30] T.E. Stringer, Plasma Phys. Controlled Fusion 33, 1715 (1991). 
Univverilty of California

Lawrence Livermere National Laboratery

Technical Information Department

Livermore, CA 94551

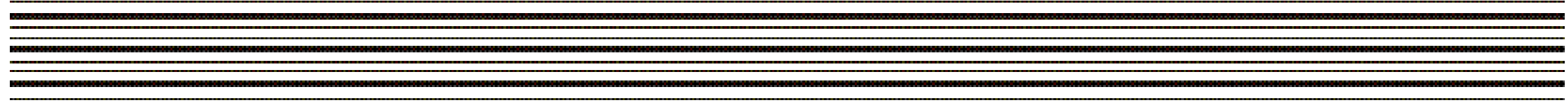

